\title{
Emerging Capabilities for Mars Exploration
}

C. D. Edwards, Jr. ${ }^{1}$, M. J. Amato ${ }^{2}$, J. D. Baker ${ }^{1}$, N. J. Barba ${ }^{1}$, J. Balaram ${ }^{1}$, E. J. Brandon ${ }^{1}$, P. Briggs $^{1}$, B. D. Davis ${ }^{3}$, J. C. Day ${ }^{1}$, A. Freeman ${ }^{1}$, M.R. Grover ${ }^{1}$, G. S. Hubbard ${ }^{4}$, D. B. Lavery ${ }^{5}$, L. Mandrake $^{1}$, L. H. Matthies ${ }^{1}$, G. Meirion-Griffith ${ }^{1}$, M. M. Munk ${ }^{6}$, I. A. Nesnas ${ }^{1}$, R. W. Obbard ${ }^{7}$, M. Ono ${ }^{1}$, M. A. Peck $^{8}$, V. Stamenković ${ }^{1}$, S. A. Townes ${ }^{1}$, T. G. Trenkle ${ }^{2}$, E. Venkatapathy ${ }^{9}$, K. A. Zacny ${ }^{10}$

${ }^{1}$ Jet Propulsion Laboratory, California Institute of Technology, ${ }^{2}$ NASA GSFC, ${ }^{3}$ NASA MSFC, ${ }^{4}$ Stanford University, ${ }^{5}$ NASA Headquarters, ${ }^{6}$ NASA LaRC, ${ }^{7}$ SETI Institute, ${ }^{8}$ The Johns Hopkins University Applied Physics Laboratory, ${ }^{9}$ NASA ARC, ${ }^{10}$ Honeybee Robotics

Contact: C. D. Edwards, Jr. (Jet Propulsion Laboratory, California Institute of Technology) email: chad-edwards@jpl.nasa.gov,phone: 818.354.4408

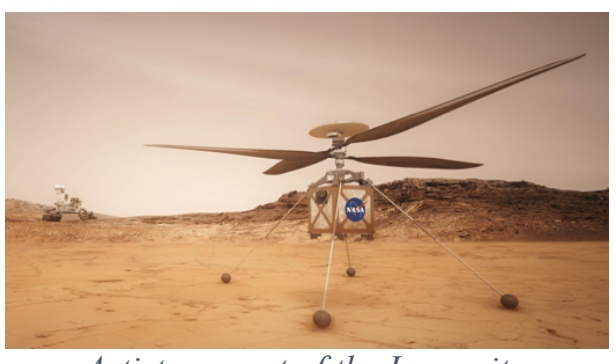

Artist concept of the Ingenuity

Helicopter demo on Mars 2020
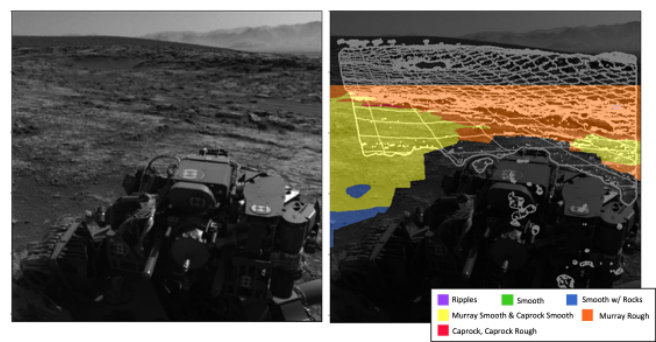

Onboard rover terrain classification for safe and energy-aware path planning

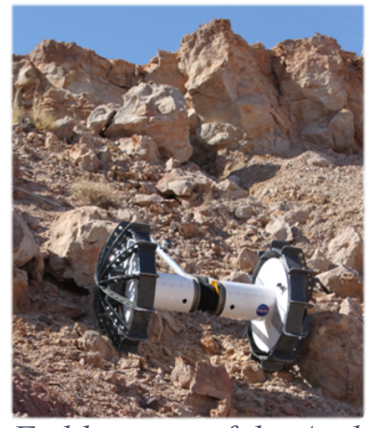

Field testing of the Axel Tethered Rover

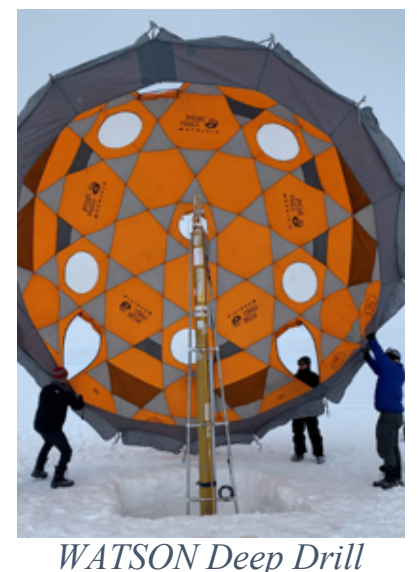

WATSON Deep Drill under test in Greenland

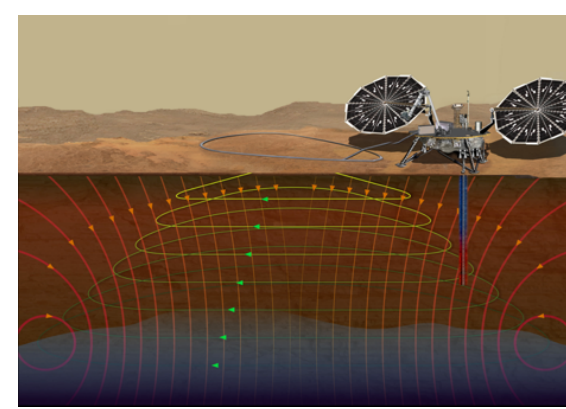

VALKYRIE subsurface mission concept

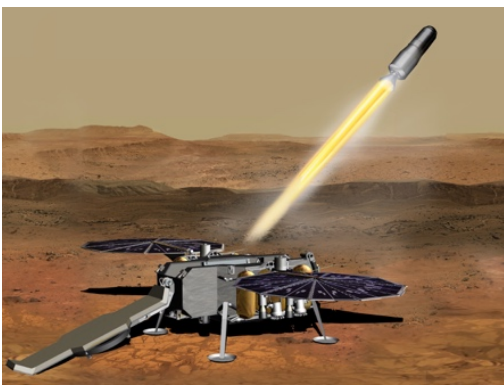

Artist concept of the MSR Mars Ascent Vehicle launch 


\section{Introduction}

Since 1997, an international series of orbiting and landed spacecraft has established a continuous robotic presence at Mars, transforming our understanding of the Red Planet. Underlying this progress has been a steady advance in our capabilities across a wide range of technologies critical for exploring Mars. The progression of Mars orbital missions has seen substantial improvements in the resolution of remote sensing instruments, coupled with large increases in communication bandwidth to accommodate the corresponding growth in science data return. Landed missions have similarly evolved, with increased landed mass, improved landing accuracy, robust mobility, enhanced sampling capabilities, and enhanced operational efficiency for in situ operations. From the tiny Sojourner Rover deployed by the Pathfinder Lander in 1997, we progressed by 2012 to the Mars Science Laboratory mission, whose Curiosity Rover has traversed over $22 \mathrm{~km}$, collecting numerous drill samples for sophisticated geochemical analysis. The Mars 2020 mission will further build on this heritage, deploying the Perseverance Rover to Jezero Crater to collect and cache samples as a possible first step in a multimission Mars Sample Return (MSR) Campaign.

The next decade of Mars exploration will demand further capability advances to address key questions of life, climate, and geology, and to lay the foundation for future human exploration. In this white paper we assess these essential capability needs across a range of technology areas, characterizing the current state of the art and identifying key capability gaps tied to high-priority mission concepts for the 2023-2032 time frame, as well as emerging technologies whose maturation in the coming decade can enable longer term Mars exploration pathways. We note that while this white paper is focused on Mars exploration mission needs, many of the identified capabilities have broader applicability to missions across the solar system.

\section{Assessment of Key Capability Areas}

\section{Entry, Descent, and Landing}

The state of the art for Mars robotic Entry Descent and Landing (EDL) systems is represented by the Mars 2020 EDL system, with heritage to the successful MSL mission that landed the 900-kg Curiosity Rover in 2012. To date all successful U.S. landings on Mars have utilized architectures closely aligned with the Viking EDL system used in 1976: a blunt body $70^{\circ}$ sphere-cone entry vehicle, a disc-gap-band supersonic parachute, and either an airbag terminal descent system or rocket-powered terminal descent system. MSL added hypersonic guidance to the previous ballistic entry systems. Mars 2020 is further advancing the state of the art by utilizing a parachute deploy range trigger and terrain-relative navigation (TRN) system to perform a more accurate landing and select a safe landing area for the first time at Mars.

In the period of this decadal survey, Mars EDL system capabilities could perform pinpoint landing ( $<100 \mathrm{~m}$ landing error) in support of the conceived Mars Sample Return (MSR) campaign. The current MSR EDL system design leverages the Mars 2020 TRN system and would incorporate a large divert during powered descent to give the lander unprecedented landing accuracy to support sample retrieval. Technologies to support pinpoint landing and hazard avoidance on the Moon, such as Navigation Doppler Lidar and Hazard Detection Lidar, are under development by NASA and can be applied at Mars. The current MSR baseline architecture is also planning enhanced supersonic parachute capability with an increase in parachute size. MSR would also utilize an innovative EDL system to ultimately return samples to Earth. To meet stringent back-planetary-protection reliability objectives, the MSR Earth Entry Vehicle would 
use neither a parachute nor retro-propulsion; rather, the passive design would employ a highly robust heatshield system based on 3-D weaving technology (HEEET) along with an impact attenuation system to maintain robust containment of the returned Martian samples.

In addition to the flagship MSR mission, smaller, lower-cost Mars landers are needed to fill key Strategic Knowledge Gaps and emplace infrastructure for the next decade. Novel concepts such as hard landers, penetrators, and low-cost surface networks that can be delivered as either ride-along assets or small missions could play a valuable role in increasing Mars surface access. Lower-cost approaches to guided entry are needed to deliver these landers closer to science targets.

To support future exploration of Mars beyond the period of the survey, EDL systems need to deliver more massive assets to scientifically interesting locations, and potentially near other landed assets, with greater precision and landing site elevation capability. Investments in deployable decelerators like Hypersonic and Supersonic Inflatable Aerodynamic Decelerators (HIAD, SIAD) and Adaptive Deployable Entry and Placement Technology (ADEPT) are needed to further extend the Viking EDL architecture. A transition from the mass-limited, Viking-derived architectures to the use of supersonic retro propulsion (SRP) will be needed to support delivering masses in the multi-ton class to the surface. SRP is also needed to enable delivery of very large payloads in support of human class missions.

\section{Surface \& Aerial Mobility}

Advances in mobility systems can offer unprecedented access to previously unexplored regions on Mars, as well as increased traverse distances to more effectively sample the diversity of Mars in situ environments. Tethered surface vehicles are now at technology readiness level (TRL) 5-6 for accessing steep slopes by rappelling down from the top, enabling access to regions of high scientific interest such as exposed ice scarps, crater walls containing recurring slope lineae, sinkholes, and caves. Rovers capable of total mission traverse distances of $\mathbf{1 0 0 - 1 0 0 0} \mathbf{~ k m}$ are conceivable in the coming decade with continued investment in miniaturized, high-performance avionics and onboard autonomy. Work is progressing toward long-lived actuators that can operate without heaters in Mars' coldest temperatures, which would reduce energy consumption and widen operational windows to facilitate mobility systems for middle and high latitudes.

Balloon and fixed wing aircraft concepts for Mars were matured in the past, but not yet flown. The 2020 Mars rover mission carries a technology demonstration of the first rotorcraft ever to go to another planet (Ingenuity, $\sim 2 \mathrm{~kg}$ total mass). Current technology development efforts aim to scale this capability to a 20 - to $30-\mathbf{k g}$ rotorcraft that could carry a payload of several kilograms and fly several kilometers per sol, depending on the site and season. This has potential for traversing very large distances, entering otherwise inaccessible terrain, and making measurements over a wide range of altitudes.

\section{Subsurface Access}

The recently revised MEPAG Goals document states that: "the subsurface should be considered an exciting new frontier for Mars exploration, and a particularly promising target environment to address the objectives presented in Goal I (Life)." It is also a key exploration target for MEPAG Goal IV (Human Exploration) as it contains resources (and potential toxins) relevant for human exploration. And access to ice in the Polar Layered Deposits offers a preserved record of Mars climate (Goal II). To date, however, the Mars subsurface has been barely explored: direct access has been limited to $5 \mathrm{~cm}$ depth in rocks by Curiosity and $10 \mathrm{~s}$ of $\mathrm{cm}$ in loose regolith by Viking/Phoenix. Four technology developments are critical to move deeper: (1) subsurface 
sounding to determine the location, availability and chemistry of liquid groundwater and ices, (2) drilling to provide ground truth for sounding and to determine geochemical and redox gradients, measure the radiation environment, and explore potential signatures of life as a function of depth, (3) sample delivery from boreholes to instruments on the surface; and (4) downhole instrumentation for subsurface in situ measurements.

Subsurface sensing by low-mass transient electromagnetic sounders can, from the surface, detect liquid groundwater to depths of kilometers and characterize the water's salinity. Orbital ground-penetrating radar is a complementary technology that can address the distribution of shallow ices, relevant not just for ISRU but also for understanding Mars' climate history; here, advancements and miniaturization in radar sounding, polarimetric and interferometric synthetic-aperture radar will be key to mapping shallow ices in the first $\sim 10-50 \mathrm{~m}$ of the subsurface.

Drilling and sampling technologies are already developed for harsh terrestrial environments. Drills to 1-10 m depths have been demonstrated under simulated Mars conditions, hundred-meter drills have been demonstrated in analog environments, and drills that can reach depths greater than $100 \mathrm{~m}$ under Mars conditions are currently under development. Mars-relevant drills like the rotary-percussive wireline Planetary Deep Drill and the rotary percussive wireline drill with deep UV/Raman spectrometer WATSON have been deployed under simulated Mars conditions and/or in Mars-analogue environments. Miniaturized wireline drilling approaches could enable drilling to depths of meters up to kilometer depths without significant changes in payload size, with targeted mass of $<50 \mathrm{~kg}$ and average power of $<100 \mathrm{~W}$, and with a design that is consistent with planetary protection protocols. The coiled tubing drill RedWater could be deployed from a Curiosity-sized rover and penetrate the subsurface to tens of meters and possibly hundreds of meters of depth, while next-generation iterations of PDD/WATSON could reach depths of $1-2 \mathrm{~km}$. Coring augers can acquire ice or rock cores for in situ micro-CT to characterize structure and layering, while deeper drills currently under development for reaching Europa oceans could also be deployed on Mars to penetrate through polar ice caps several kilometers deep. Across all drill types, new downhole instrumentation and/or sampling systems to convey drill samples to more capable lander instruments will be high priorities.

\section{Autonomy}

Autonomy - the ability of a system to make and act on internal decisions to achieve goals - is critical to addressing two fundamental challenges for Mars exploration: communication cadence and bandwidth. Limited communication cadence (i.e., once-per-sol command cycle) has strong implications for rover surface operations productivity and safety, while limited bandwidth heavily impacts overall science return. Over the past decade, several key onboard capabilities have begun to emerge with significant promise to expand the boundaries of Mars exploration and increase science yield, mitigating mission risk and enabling fundamentally new orbital and surface mission concepts. Engineering self-sufficiency, enabled in part by technologies such as the onboard planner deployed on Mars 2020, allows a vehicle to make informed choices about what is possible and safe, given current in situ knowledge, for both nominal and off-nominal scenarios. Onboard change detection algorithms on future orbiters can identify change on scales of days to months to capture, characterize, and map new science targets and their distribution in time and space. Onboard target recognition systems (e.g., WATCH, AEGIS) can identify scientifically interesting targets based on past observations, informing onboard compression, prioritization, and reactive observation systems to ensure these targets are preferentially captured. Anomaly 
detection systems operate in reverse of target recognition, seeking to capture and characterize findings from observations that are distinctly different from an onboard model of normalcy. Onboard knowledge-based compression algorithms, designed to recognize both known and anomalous science targets along with justifications for decisions made, have the ability to reduce copious data volumes into highly efficient summary products.

EDL's rapid timeline demands high levels of autonomy to achieve safe and precise landing, based on terrain-relative navigation, landing hazard assessment, and fuel-efficient divert maneuvers. Once on the surface, mobile ground and aerial platforms will require advanced autonomy to support fast ( $>1 \mathrm{~km} / \mathrm{sol}$ ) traverse capabilities. Tethered surface navigation enables safe and long traverses per sol in steep and rocky terrains. Terrain classification and related algorithms enable assessment of hazards for different mobility modalities, including geometric, non-geometric (e.g., sand traps), fall (for tethered rovers), and landing (for aerial platforms). Riskand resource-aware path planning enables generation and selection of drive/flight routes with explicit consideration of local resource (e.g., energy, comm relay) and safety constraints. Absolute self-localization against orbital maps maintains global position knowledge over long traverses.

Autonomy applications in flight are today limited by the current set of low-performance space-qualified processors (e.g. MIL-STD-1750A, RAD6000, RAD750). To enable the next era of Mars exploration, higher computing power is needed to support increased autonomy applications, as well as smaller, lower-power solutions for small spacecraft. Development in the following areas will enable more challenging missions, increase science return, and speed the evolution of autonomy applications: 1) Distributed processing architectures to focus processing resources on specific tasks, shared information and high availability; 2) Size-, mass-, and poweroptimized, high-reliability avionics processor platforms for basic vehicle functions, instruments and low-bandwidth autonomy as well as specialty rovers, probes and small satellites; 3) High-performance, multi-core processing platforms to address advanced autonomy needs like adaptive optics, hazard assessment and avoidance, spectral data processing and autonomous science; 4) Software, behaviors and infrastructure that allow implementers to focus on more complex, mission-specific developments and encourage partnerships with universities and industry. Infusion of commercial processor technology offers a path to exceptional performance at low mass/power, as evidenced by the Qualcomm Snapdragon processor on the Ingenuity helicopter.

\section{Communications \& Navigation}

Communication and navigation support for Mars Exploration continues to evolve, both for Mars-Earth and Mars-local connections. For Mars-Earth links, the DSN will have one 70m antenna and four $34 \mathrm{~m}$ BWG antennas at each complex with S-, X-, and Ka-band capabilities. Any combination of antennas at a complex can be arrayed for more receive aperture or higher uplink power. Multiple Spacecraft Per Aperture capabilities will allow a single DSN antenna to support up to four simultaneous downlinks, with agile sharing of the uplink, enabling efficient support to the increasing number of spacecraft at Mars. Tracking capabilities are being extended/improved to include regenerative ranging and wider-bandwidth $\triangle \mathrm{DOR}$.

At Mars, communication relays will continue to play a key role in increasing data return from Mars - ideally continuing the policy of including a relay payload on each orbiting mission. Multi-band, multiuser, software-defined radios will be the norm. Future Mars proximity links can include X-band along with UHF for increased science return. Steered higher-gain X-band antennas on both surface and orbiting assets will improve data rates on these links. A robust orbital 
relay network will enable low-cost small satellite missions to achieve MRO-class data return without the need for their own costly direct-to-Earth comm links. Tying all of this together will be a network-based approach utilizing Delay-Tolerant Networking to provide automated assured delivery of products between PIs and their instruments. Finally, toward the end of the decade, optical communication will be coming online as the DSN and our international partners deploy large-aperture optical ground receivers (e.g., DSN Hybrid RF-Optical 34m BWG antenna), and flight telecom systems include both RF and optical capabilities.

\section{Power}

Power systems will continue to play a critical role in future Mars exploration campaigns. Advanced mechanical and electrostatic dust mitigation technologies are under development for integration with high-performance, lightweight deployable solar arrays. These new capabilities will address the perennial issue of dust coverage on solar arrays and reduce the uncertainties associated with wind removal.

For continuous operations through the night and to support long-duration polar missions, an "enhanced" version of the current Multi-Mission Radioisotope Thermoelectric Generator (MMRTG) is under development. This proposed eMMRTG (targeted for TRL 6 by 2026) would be capable of operating in the atmosphere of Mars and will feature a specific power $\sim 17 \%$ higher than current MMRTG technology ( $\sim 133 \mathrm{~W}$ at launch). A lower-TRL, higher-performing, scalable option (NextGen RTG) is also under development, offering a modular design with output power options spanning the range of 50-400 W. However, based on NASA's Radioisotope Power Source technology development roadmap, this design is currently only applicable to vacuum operations; re-design for compatibility with Mars surface applications should be a high agency priority.

Both solar and RTG power sources would be used with a range of emerging energy storage options to support peak power requirements, load levelling and night operations. Improved high specific energy $(>300 \mathrm{Wh} / \mathrm{kg})$, rechargeable lithium-ion cells that can operate over wide temperature ranges $\left(-30^{\circ} \mathrm{C}\right.$ and below) are under development. High power $(>\mathbf{1} \mathbf{~ k W} / \mathbf{k g})$ rechargeable batteries are targeted to replace one-time-use thermal batteries, enhancing the reliability of critical EDL systems, supporting high-power drilling operations, and extending flight times for future Mars rotorcraft, but will require further investments to meet aggressive power, energy and thermal requirements. In addition, significantly improved primary battery technologies are under development for supporting small probes and hard landers, with options for very high specific energy $(>700 \mathrm{Wh} / \mathrm{kg})$ and ultra-low temperature $\left(<-80^{\circ} \mathrm{C}\right)$ operation. To support more challenging robotic surface exploration, advanced power transfer techniques are being developed, including robust, efficient tethers and compact laser power beaming systems, allowing larger power sources such as fixed solar arrays or RTGs to "extend" their range to small, highly mobile robotic systems.

\section{Propulsion}

Many state-of-the-art (SOA) propulsion capabilities are being deployed in the conceptual Mars Ascent Vehicle (MAV) system as part of a Mars Sample Return (MSR) campaign. The current MAV baseline design features two solid rocket motor stages with cold-capable carboxyl terminated polybutadiene propellant, composite motor cases, trapped ball nozzles with supersonic split lines, thrust vector control. Attitude and roll control as well as axial orbital trim thrust would be provided by a hydrazine monopropellant reaction control system. Prior to selection of the solid 
propellant option, technology investments also advanced the readiness of a hybrid propellant option for the MAV, demonstrating new low-temperature fuel formulations and new solid fuel hypergolic ignition technology, positioning hybrid as a safe, high-performance low-cost solution for future low-temperature operations.

"Green" propellants, such as AF-M315E, have much lower temperature capability, higher volumetric density, and have been recently demonstrated in orbit. Further development of green monopropellants and compatible thrusters could enable future propulsion systems that require less power for thermal conditioning and are safer to handle on Earth, thereby reducing costs. Additively manufactured composite structures could enable more efficient production of future propulsion systems, with significant reductions in cost, mass, and schedule. For larger sized stages, including human rated vehicles, cryogenic bipropellant systems offer increased performance. These systems would benefit from emerging technologies including liquid oxygen/methane (LOx/LCH4) engines, cryogenic fluid management, and in-situ resource utilization. The Integrated Cryogenics for Exploration (ICE) project is attempting to leverage the above areas of focus with the addition of pulsed pump-fed cryogenic thrusters and shared main propulsion and RCS fuel tanks.

\section{Planetary Protection}

The return of samples from the Martian surface will be contingent on the successful development of technologies for highly robust containment of those samples, to prevent the unintended release of any unsterilized Mars particles into the Earth biosphere. Current MSR plans call for breaking the chain of contact with Mars and providing containment assurance utilizing brazing techniques which can create a mechanical seal for a sample containment vessel while simultaneously sterilizing the seal.

Future astrobiologically driven mission concepts, including searches for modern habitable environments and extant life in the Martian subsurface, will require stringent contamination control and sterilization techniques to minimize the forward contamination of those environments with Earth-based organic material or life forms. Spacecraft component designs compatible with Dry Heat Microbial Reduction and Vaporized Hydrogen Peroxide sterilization techniques, combined with bio-barrier technologies to maintain aseptic conditions, will be key to meeting these challenges.

\section{Small Spacecraft Technologies}

The Mars Cubesat One (MarCO) mission demonstrated the potential for small, low-cost satellites to successfully perform interplanetary missions. Future Small Spacecraft (SSc) offer a pathway to highly affordable, compelling Mars missions that could be implemented in parallel with Mars Sample Return, addressing key science questions, delivering essential infrastructure, and providing reconnaissance for future human exploration activities. Multi-element networks of SSc at Mars present a unique opportunity to acquire diverse spatiotemporal observations of Mars global processes. SSc missions can play an integral role as pathfinders for, or to augment, future larger-scale missions and campaigns, and encourage collaboration from partners in academia and other institutions around the world.

Recent mission studies demonstrate that SSc can deliver compelling Mars science at a small fraction of Discovery mission costs. With total mission costs of \$100M-\$300M, SSc missions can deliver $\sim 20 \mathrm{~kg}$ of science payload to Mars orbit, while SSc hard landers can deliver up to $\sim 6 \mathrm{~kg}$ of science payload to the Martian surface at or under that same cost 
target. Delivery options include riding as payload on a dedicated Mars-bound mission, leveraging commercial rideshare options, or using emerging small-launch vehicles to reach Earth orbit and then using low-cost propulsion to complete the transfer to Mars. New miniaturized instruments and avionics systems for deep space will enable SSc mission concepts. Deployable drag skirts, using technologies such as ADEPT, can enable aerocapture for SSc orbiters and increase delivered payload mass for launch-volume-constrained SSc landers. A deeper examination of the science, implementation, technology, applications, and policies enabling small spacecraft exploration at Mars can be found in the separate white paper "High Science Value Return of Small Spacecraft at Mars" (Barba, et al.)

\section{Conclusions}

Advances in the areas identified here will set the stage for the next decade of Mars exploration. A Mars Ascent Vehicle, representing the first launch from another planet, robust Containment Assurance technologies, and further evolution of our EDL capabilities will enable the first-ever return of samples from the Martian surface. New mobility systems, including long-range and extreme terrain rovers as well as capable rotorcraft, will provide access to diverse and previously unexplored regions. New remote sensing and drilling technologies will grant entry to, and characterization of, the Martian subsurface, the most likely abode for modern habitable environments on Mars. Advances in autonomy, enabled by more capable flight processors, will facilitate operation in challenging in situ environments; coupled with higherbandwidth Mars-Earth communication capabilities, future missions will achieve higher productivity and increased science return. Progress in power and propulsion technologies will benefit a range of new Mars mission concepts. And new Small Spacecraft technologies, coupled with innovative launch options, will make possible decadal-class science missions at unprecedented low cost.

Achieving these goals will require focused NASA technology investments tied to welldefined science objectives, coupled with pro-active infusion of emerging commercial technologies and coordination with other government agencies. Ultimately, these advances will provide the critical mission capabilities needed to expand our understanding of the Red Planet in the decades ahead.

\section{References}

A complete set of bibliographic references is available at:

https://docs.google.com/document/d/1Dwc5ii3XQGnWe etJNuW7epx7Lvxbzm19ALH5IiQUPc ledit?usp=sharing

The information in this document is pre-decisional and is provided for planning and discussion only. The cost information contained in this document is of a budgetary and planning nature and is intended for informational purposes only. It does not constitute a commitment on the part of JPL and/or Caltech.

A portion of this research was carried out at the Jet Propulsion Laboratory, California Institute of Technology, under a contract with the National Aeronautics and Space Administration (80NM0018D0004). 